\title{
Global well-posedness of strong solution for the three dimensional dynamic Cahn-Hilliard-Stokes model
}

\author{
Kelong Cheng ${ }^{\mathrm{a}, *}$, Wenqiang Feng ${ }^{\mathrm{b}}$ \\ a School of Science, Southwest University of Science and Technology, Mianyang, Sichuan 621010, China. \\ ${ }^{b}$ Department of Mathematics, University of Tennessee, Knoxville, Tennessee 37996, USA. \\ Communicated by S. S. Chang
}

\begin{abstract}
The global well-posedness analysis for the three dimensional dynamic Cahn-Hilliard-Stokes (CHS) model is provided in this paper. In this model, the velocity vector is determined by the phase variable by both the Darcy law and the Stokes equation. Based on the analysis of weak solutions to the CHS equation by the standard Galerkin method, we present a global in time strong solution for the CHS model. Moreover, the existence and the uniqueness of the strong solution are also proven. (C)2017 All rights reserved.
\end{abstract}

Keywords: Cahn-Hilliard-Stokes model, Sobolev embedding, Galerkin procedure, Hemlholtz projection. 2010 MSC: 35K55, 76D05.

\section{Introduction}

The Cahn-Hilliard equation [3] plays an important role in the mathematical study of the spontaneous separation of binary fluid, and has attracted much attention both analytically and numerically (see $[1,2,6-8,10,11,16-18]$ and the references therein). Recently, the couplings of the Cahn-Hilliard equation with other basic modeling equations also have been proposed in various situation to describe complicated phenomena in fluid mechanics involving phase transition, such as the Cahn-Hilliard-Navier-Stokes (CHNS) equation [5, 9, 13, 14, 19], the Cahn-Hilliard-Hele-Shaw (CHHS) [4, 12, 22-24] equation, and the Cahn-Hilliard-Boussinesq (CHB) [26] equation.

The Cahn-Hilliard energy of a binary fluid with a constant mass density is given by

$$
\mathrm{E}_{\mathrm{CH}}(\phi)=\int_{\Omega}\left\{\frac{1}{4} \phi^{4}-\frac{1}{2} \phi^{2}+\frac{\epsilon^{2}}{2}|\nabla \phi|^{2}\right\} \mathrm{d} \mathbf{x},
$$

where $\Omega \subset \mathbf{R}^{3}, \phi: \Omega \rightarrow \mathbf{R}$ is the concentration field, and $\epsilon$ is the interfacial thickness parameter.

\footnotetext{
*Corresponding author

Email addresses: zhengkelong@swust.edu.cn (Kelong Cheng), wfeng1@vols.utk.edu (Wenqiang Feng) doi:10.22436/jnsa.010.08.16
} 
The phase equilibria are represented by the pure phase $\phi= \pm 1$. For simplicity, we assume that $\Omega=$ $\left(0, L_{x}\right) \times\left(0, L_{y}\right) \times\left(0, L_{z}\right)$ and that $\partial_{\mathfrak{n}} \phi=0$ on $\partial \Omega$, the latter condition representing local thermodynamic equilibrium on the boundary. In this paper, we discuss dynamic equations for the Cahn-Hilliard-Stokes (CHS) model as follows,

$$
\begin{aligned}
& \partial_{\mathrm{t}} \phi+\mathbf{u} \cdot \nabla \phi=\Delta \mu, \\
& \partial_{\mathrm{t}} \mathbf{u}-\Delta \mathbf{u}+\mathbf{u}+\nabla p=-\gamma \phi \nabla \mu, \\
& \nabla \cdot \mathbf{u}=0
\end{aligned}
$$

where the chemical potential is defined as

$$
\mu:=\delta_{\phi} E=\phi^{3}-\phi-\epsilon^{2} \Delta \phi,
$$

and $\boldsymbol{u}$ is the advective velocity and $p$ is the pressure. We assume free-slip boundary conditions for the velocity field, namely $\mathbf{u} \cdot \mathfrak{n}=0, \frac{\partial \mathbf{u} \cdot \boldsymbol{\tau}}{\partial \mathfrak{n}}=0$ and no-flux boundary condition for $\mu, \partial \mathfrak{n} \mu=0$ on $\partial \Omega$.

The total energy of dynamic equations for the CHS model is given by

$$
\mathrm{E}(\phi, \mathbf{u})=\int_{\Omega}\left\{\frac{1}{4} \phi^{4}-\frac{1}{2} \phi^{2}+\frac{\epsilon^{2}}{2}|\nabla \phi|^{2}+\frac{1}{2 \gamma}|\mathbf{u}|^{2}\right\} \mathrm{d} \mathbf{x}=\mathrm{E}_{\mathrm{CH}}(\phi)+\frac{1}{2 \gamma}\|\mathbf{u}\|_{\mathrm{L}^{2}}^{2},
$$

with $\mathrm{E}_{\mathrm{CH}}(\phi)$ given by (1.1). The system (1.2), (1.3), (1.4) is mass conservative and energy dissipative, and the dissipation rate is readily found to be

$$
\mathrm{d}_{\mathrm{t}} \mathrm{E}=-\int_{\Omega}|\nabla \mu|^{2} \mathrm{~d} \mathbf{x}-\frac{1}{\gamma} \int_{\Omega}\left(|\nabla \mathbf{u}|^{2}+|\mathbf{u}|^{2}\right) \mathrm{d} \mathbf{x} \leqslant 0
$$

Although there exist a great deal of theoretical analysis and numerical methods investigating the CahnHilliard equation and the Stokes (or other variations) equation, respectively, it still brings some additional technical difficulties for these new coupled systems in theoretical and numerical aspects. We now present several results which are especially relevant to this paper. In [20], an initial-boundary value problem for the Cahn-Hilliard-Hele-Shaw system that models tumor growth is studied. For large initial data with finite energy, the authors proved global existence, uniqueness, higher order spatial regularity, and Gevrey spatial regularity of strong solutions in 2-3D. Asymptotically in time, it is shown that the solution converges to a constant state exponentially fast as time tends to infinity under certain assumptions. The well-posedness of the Darcy-Cahn-Hilliard model for the Hele-Shaw flow as well as the basic regularity of the weak solution can be found in [12]. In more detail, a convex splitting numerical scheme was formulated, with a mixed finite element approximation in space. Such an approximate construction assures an unconditional energy stability. As a result, using certain compactness arguments, the authors obtained a weak convergence of the finite element numerical approximation to a global-in-time weak solution. Furthermore, the CHHS solution with higher order regularities is discussed in [23] by more advanced Littlewood-Paley theory, and global in time classical solutions were reported for the 3-D CHHS system in [22], if the initial data is close to an energy minimizer or the Péclet number is sufficiently small.

In this paper, the global well-posedness analysis of the CHS equation is mainly accomplished by an $\mathrm{L}^{\infty}$ estimate of the velocity variable in terms of an $\mathrm{L}^{2}$ norm of $-\gamma \phi \nabla \mu$, which comes from an application of elliptic regularity and Hemlholtz projection to the Stokes equation (1.3). With the estimates for the nonlinear terms, a uniform in time $\mathrm{H}^{2}$ estimate for the phase variable can be obtained. Consequently, a standard Galerkin procedure would construct approximate solutions and the limit functions turns out to be the unique strong solution of the CHS system.

The remainder of the paper is organized as follows. In Section 2, we review and analyze the weak solution to the dynamic CHS equation. In Section 3, a global in time strong solution, with regularity of $\mathrm{L}^{\infty}\left(0, \mathrm{~T} ; \mathrm{H}^{2}\right) \cap \mathrm{L}^{2}\left(0, \mathrm{~T} ; \mathrm{H}^{4}\right)$ is established for the CHS system (1.2), (1.3), (1.4), with any $\mathrm{H}^{2}$ initial data for $\phi$. Furthermore, the existence and uniqueness of the strong solution are also proven. 


\section{Global in time of the weak solution}

2.1. Review of the weak solution

For any positive final time $T$, the functions $(\phi, \mathfrak{u})$ with the following regularities

$$
\begin{aligned}
& \phi \in \mathrm{L}^{\infty}\left(0, \mathrm{~T} ; \mathrm{H}^{1}\right) \cap \mathrm{L}^{2}\left(0, \mathrm{~T} ; \mathrm{H}^{3}\right), \quad \partial_{\mathrm{t}} \phi \in \mathrm{L}^{1}\left(0, \mathrm{~T} ; \mathrm{H}^{-1}\right) \cap \mathrm{L}^{2}\left(0, \mathrm{~T} ; \mathrm{H}^{-3}\right), \\
& \mathbf{u}=(\mathrm{u}, v, w) \in \mathrm{L}^{\infty}\left(0, \mathrm{~T} ; \mathrm{L}^{2}\right) \cap \mathrm{L}^{2}\left(0, \mathrm{~T} ; \mathrm{H}^{1}\right),
\end{aligned}
$$

are called a weak solution for the dynamic CHS system (1.2), (1.3), (1.4), if

$$
\begin{aligned}
& \left(\partial_{\mathrm{t}} \phi, \psi\right)-(\mathbf{u} \phi, \nabla \psi)=(\mu, \Delta \psi), \quad \forall \psi \in \mathrm{H}^{3}(\Omega), \\
& \partial_{\mathrm{t}} \mathbf{u}-\Delta \mathbf{u}+\mathbf{u}=-\gamma \mathcal{P}_{\mathrm{H}}(\phi \nabla \mu),
\end{aligned}
$$

where $\mu=\phi^{3}-\phi-\varepsilon^{2} \triangle \phi$ and $\mathcal{P}_{H}$ denotes the standard Helmholtz projection, which leads to $\mathcal{P}_{H} \nabla p=0$.

Theorem 2.1. Let $\phi_{0}=\phi(\cdot, 0) \in \mathrm{H}^{1}(\Omega)$. Then there exists at least one global weak solution for the dynamic CHS equation (1.2), (1.3), (1.4), such that for any $\mathrm{T}>0$

$$
\begin{aligned}
& \|\phi\|_{\mathrm{L}^{\infty}\left(0, \mathrm{~T} ; \mathrm{H}^{1}\right)} \leqslant A_{1}, \quad\|\phi\|_{\mathrm{L}^{2}\left(0, \mathrm{~T} ; \mathrm{H}^{3}\right)} \leqslant A_{2}, \quad\left\|\partial_{\mathrm{t}} \phi\right\|_{\mathrm{L}^{2}\left(0, \mathrm{~T} ; \mathrm{H}^{-3}\right)} \leqslant A_{3}, \\
& \|\boldsymbol{u}\|_{\mathrm{L}^{\infty}\left(0, \mathrm{~T} ; \mathrm{L}^{2}\right)} \leqslant A_{4}, \quad\|\boldsymbol{u}\|_{\mathrm{L}^{2}\left(0, \mathrm{~T} ; \mathrm{H}^{1}\right)} \leqslant A_{5},
\end{aligned}
$$

where $A_{1}$ and $A_{4}$ are time independent and $A_{2}, A_{3}$, and $A_{5}$ are time dependent constants.

Before we prove this theorem, we build some framework.

\subsection{Construction of approximation solution and properties}

To prove Theorem 2.1, we recall the following Galerkin procedure.

Let $\left\{\Phi_{j}\right\}_{j} \geqslant 1 \subset H^{2}(\Omega)$ be eigenfunctions of the Laplacian operator corresponding to the eigenvalues $-\lambda_{j}, j=1,2, \cdots$, with $0<\lambda_{1}<\lambda_{2}<\cdots$, such that

$$
-\triangle \Phi_{j}=\lambda_{j} \Phi_{j},\left.\quad \frac{\partial \Phi_{j}}{\partial \mathfrak{n}}\right|_{\partial \Omega}=0
$$

Additionally, we choose $\left\|\Phi_{j}\right\|=1$ for $j \geqslant 1$. Obviously, $\left\{\Phi_{j}\right\}_{j} \geqslant 1 \subset C^{\infty}(\Omega)$ defines a complete orthonormal basis in $\mathrm{L}^{2}(\Omega)$.

Denote $G_{M}$ as the vector space spanned by $\left\{\Phi_{j}\right\}_{j=1}^{M}$ and define $\mathcal{P}_{M}$ as the standard projection from $\mathrm{L}^{2}(\Omega)$ to $\mathrm{G}_{\mathrm{M}}$ :

$$
\mathcal{P}_{M}=\sum_{j=1}^{M} \hat{f}_{j} \Phi_{j}, \quad \text { for } \quad f=\sum_{j=1}^{\infty} \hat{f}_{j} \Phi_{j}, \quad \hat{f}_{j}=\left(f, \Phi_{j}\right) .
$$

To seek a weak solution of the CHS equation (1.2), (1.3), (1.4), we find an approximate solution $\left\{\phi_{M}, \overline{\mathbf{u}}_{M}\right\}$ with $\phi_{M} \in G_{M}$, such that

$$
\begin{aligned}
& \partial_{t} \phi_{M}+\mathcal{P}_{M}\left(\overline{\mathbf{u}}_{M} \cdot \nabla \phi_{M}\right)=\Delta \mu_{M}, \\
& \partial_{t} \overline{\mathbf{u}}_{M}-\triangle \overline{\mathbf{u}}_{M}+\overline{\mathbf{u}}_{M}=-\gamma \mathcal{P}_{H}\left(\phi_{M} \nabla \mu_{M}\right), \\
& \left.\phi_{M}\right|_{t=0}=\mathcal{P}_{M} \phi_{0},
\end{aligned}
$$

where $\mu_{M}=\mathcal{P}_{M}\left(\phi_{M}^{3}\right)-\phi_{M}-\varepsilon^{2} \triangle \phi_{M} \in G_{M}$. Note that $\overline{\mathbf{u}}_{M} \notin \mathrm{G}_{M}$ in general, since $\phi_{M} \nabla \mu_{M} \notin \mathrm{G}_{M}$.

We also present the following results, which will be used in the analysis later in the paper. For brevity, in the following, $\|\cdot\|$ denotes the $\mathrm{L}^{2}$-norm $\|\cdot\|_{\mathrm{L}^{2}}$. 
Lemma 2.2. The following estimates are valid.

$$
\begin{aligned}
& \left\|\mathcal{P}_{M} f\right\| \leqslant\|f\|, \quad \forall f \in L^{2}(\Omega), \quad M \geqslant 1, \\
& \left(\mathcal{P}_{M} f, g_{M}\right)=\left(f, g_{M}\right), \quad \forall f \in L^{2}(\Omega), \quad M \geqslant 1, \quad g_{M} \in G_{M}, \\
& \left(\nabla \Phi_{i}, \nabla \Phi_{j}\right)=0, \quad \text { for } i \neq j, \\
& \left\|\nabla\left(\mathcal{P}_{M} f\right)\right\| \leqslant\|\nabla f\|, \text { for } f \text { smooth enough. }
\end{aligned}
$$

Proof.

(1) Based on the eigenfunction expansion (2.2) and its projection, we apply the orthonormal property of $\left\{\Phi_{\mathfrak{j}}\right\}_{j=1}^{\infty}$ and get

$$
\left\|\mathcal{P}_{M} f\right\|^{2}=\sum_{j=1}^{M}\left|\hat{f}_{j}\right|^{2} \leqslant\|f\|^{2}=\sum_{j=1}^{\infty}\left|\hat{f}_{j}\right|^{2}
$$

so that (2.6) is proven.

(2) For $g_{M} \in G_{M}$, assume its eigenfunction expansion is $g_{M}=\sum_{j=1}^{M} \hat{g}_{j} \Phi_{j}$. In turn, the following derivation could be made,

$$
\left(f-\mathcal{P}_{M} f, g_{M}\right)=\left(\sum_{j=M+1}^{\infty} \hat{f}_{j} \Phi_{j}, \sum_{j=1}^{M} \hat{g}_{j} \Phi_{j}\right)=0,
$$

in which the last step comes from the orthogonal property of $\left\{\Phi_{j}\right\}_{j=1}^{\infty}$.

(3) Due to the homogeneous Neumann boundary condition, (2.8) is a direct consequence of integration by parts,

$$
\left(\nabla \Phi_{i}, \nabla \Phi_{j}\right)=-\left(\Phi_{i}, \Delta \Phi_{j}\right)=-\lambda_{j}\left(\Phi_{i}, \Phi_{j}\right)=0, \quad \text { for } i \neq j .
$$

(4) The eigenfunction expansion (2.2) and its projection indicate that

$$
\nabla\left(\mathcal{P}_{M} f\right)=\sum_{j=1}^{M} \hat{f}_{j} \nabla \Phi_{j}, \quad \nabla f=\sum_{j=1}^{\infty} \hat{f}_{j} \nabla \Phi_{j} .
$$

Hence, we apply the orthogonal property (2.8) for $\left\{\nabla \Phi_{j}\right\}_{j=1}^{\infty}$ and arrive at

$$
\left\|\nabla\left(\mathcal{P}_{M} f\right)\right\|^{2}=\sum_{j=1}^{M}\left|\hat{f}_{j}\right|^{2}\left\|\nabla \Phi_{j}\right\|^{2} \leqslant\|\nabla f\|^{2}=\sum_{j=1}^{\infty}\left|\hat{f}_{j}\right|^{2}\left\|\nabla \Phi_{j}\right\|^{2},
$$

which in turn completes the proof of (2.9).

Moreover, a detailed calculation shows that the following formulas of integration by parts are also valid.

$$
\begin{aligned}
& \left(\mathrm{f}, \triangle \mathrm{g}_{M}\right)=-\left(\nabla \mathrm{f}, \nabla \mathrm{g}_{M}\right), \quad\left(\mathrm{f}, \triangle^{2} \mathrm{~g}_{M}\right)=\left(\triangle \mathrm{f}, \triangle \mathrm{g}_{M}\right), \\
& \left(\triangle \mathrm{f}, \triangle^{2} \mathrm{~g}_{M}\right)=-\left(\nabla \triangle \mathrm{f}, \nabla \triangle \mathrm{g}_{M}\right),
\end{aligned}
$$

for $g_{M} \in G_{M}$ and $f$ smooth enough.

\subsection{Proof of Theorem 2.1}

It is straightforward to obtain the local in time existence of the approximation solution, since the Galerkin scheme (2.3) and (2.5) with the velocities determined by (2.4) is an initial value problem for a system of ODEs. But to discuss the global in time of the weak solution, energy estimates are necessary. 
Proof of Theorem 2.1. Taking the inner product of (2.3) with $\mu_{M}=\mathcal{P}_{M}\left(\phi_{M}^{3}\right)-\phi_{M}-\varepsilon^{2} \triangle \phi_{M}$, by the abovementioned result (2.7) and the integration by parts formula (2.10), we have

$$
\left(\partial_{t} \phi_{M}, \mu_{M}\right)+\left(\overline{\mathbf{u}}_{M} \cdot \nabla \phi_{M}, \mu_{M}\right)+\left\|\nabla \mu_{M}\right\|^{2}=0 .
$$

For the first term, according to (2.7), $\left(\partial_{t} \phi_{M}, \mathcal{P}_{M}\left(\phi_{M}^{3}\right)\right)=\left(\partial_{t} \phi_{M}, \phi_{M}^{3}\right)$ and a direct result can be derived as follows,

$$
\begin{aligned}
\left(\partial_{t} \phi_{M}, \mu_{M}\right) & =\left(\partial_{t} \phi_{M}, \mathcal{P}_{M}\left(\phi_{M}^{3}\right)-\phi_{M}-\varepsilon^{2} \triangle \phi_{M}\right) \\
& =\left(\partial_{t} \phi_{M}, \phi_{M}^{3}-\phi_{M}-\varepsilon^{2} \triangle \phi_{M}\right) \\
& =\frac{d}{d t} E_{C H}\left(\phi_{M}\right),
\end{aligned}
$$

where $E_{C H}\left(\phi_{M}\right):=\int_{\Omega}\left[\frac{1}{4} \phi_{M}^{4}-\frac{1}{2} \phi_{M}^{2}+\frac{\varepsilon^{2}}{2}\left|\nabla \phi_{M}\right|^{2}\right] d x$. For the second term,

$$
\begin{aligned}
\left(\overline{\mathbf{u}}_{M} \cdot \nabla \phi_{M}, \mu_{M}\right) & =\left(\nabla \cdot\left(\overline{\mathbf{u}}_{M} \phi_{M}\right), \mu_{M}\right)=-\left(\overline{\mathbf{u}}_{M} \phi_{M}, \nabla \mu_{M}\right) \\
& =-\left(\overline{\mathbf{u}}_{M}, \phi_{M} \nabla \mu_{M}\right)=-\left(\overline{\mathbf{u}}_{M},-\frac{1}{\gamma}\left(\partial_{t} \overline{\mathbf{u}}_{M}-\triangle \overline{\mathbf{u}}_{M}+\overline{\mathbf{u}}_{M}\right)\right) \\
& =\frac{1}{\gamma}\left(\overline{\mathbf{u}}_{M}, \partial_{t} \overline{\mathbf{u}}_{M}-\triangle \overline{\mathbf{u}}_{M}+\overline{\mathbf{u}}_{M}\right) \\
& =\frac{1}{\gamma}\left(\frac{1}{2} \frac{d}{d t}\left\|\overline{\mathbf{u}}_{M}\right\|^{2}+\left\|\nabla \overline{\mathbf{u}}_{M}\right\|^{2}+\left\|\overline{\mathbf{u}}_{M}\right\|^{2}\right) .
\end{aligned}
$$

Substituting (2.12) and (2.13) into (2.11), we obtain the energy estimate

$$
\frac{\mathrm{d}}{\mathrm{dt}} \mathrm{E}\left(\phi_{M}\right)+\left\|\nabla \mu_{M}\right\|^{2}+\frac{1}{\gamma}\left(\left\|\nabla \overline{\mathbf{u}}_{M}\right\|^{2}+\left\|\overline{\mathbf{u}}_{M}\right\|^{2}\right)=0,
$$

in which $\mathrm{E}\left(\phi_{M}\right)=\mathrm{E}_{\mathrm{CH}}\left(\phi_{M}\right)+\frac{1}{2 \gamma}\left\|\overline{\mathbf{u}}_{M}\right\|^{2}$.

As a result, this energy bound gives a uniform in time $\mathrm{H}^{1}$ bound for the approximate solution $\phi_{M}$. In turn, a global (in time) solution for (2.3), (2.4), (2.5) is assured, for any fixed $M>1$.

Moreover, more detailed Sobolev analysis indicate the following bounds,

$$
\begin{aligned}
& \left\|\phi_{M}\right\|_{L^{\infty}\left(0, T ; H^{1}\right)} \leqslant A_{1}, \quad\left\|\phi_{M}\right\|_{L^{2}\left(0, T ; H^{3}\right)} \leqslant A_{2}, \quad\left\|\partial_{t} \phi_{M}\right\|_{L^{2}\left(0, T ; H^{-3}\right)} \leqslant A_{3}, \\
& \left\|\overline{\mathbf{u}}_{M}\right\|_{L^{\infty}\left(0, T ; L^{2}\right)} \leqslant A_{4}, \quad\left\|\overline{\mathbf{u}}_{M}\right\|_{L^{2}\left(0, T ; H^{1}\right)} \leqslant A_{5},
\end{aligned}
$$

where the constant $A_{1}$ and $A_{4}$ are time independent, $A_{2}, A_{3}$ and $A_{5}$ are time dependent. We leave to be confirmed the details to interested readers.

Since these estimates are uniform in $M$, there exist subsequences $\phi_{l M}, \bar{u}_{l M}, \partial_{t} \phi_{l M}$ and limit functions $\phi \in \mathrm{L}^{\infty}\left(0, \mathrm{~T} ; \mathrm{H}^{1}\right) \cap \mathrm{L}^{2}\left(0, \mathrm{~T} ; \mathrm{H}^{3}\right), \partial_{\mathrm{t}} \phi \in \mathrm{L}^{2}\left(0, \mathrm{~T} ; \mathrm{H}^{-3}\right), \mathbf{u} \in \mathrm{L}^{\infty}\left(0, \mathrm{~T} ; \mathrm{L}^{2}\right) \cap \mathrm{L}^{2}\left(0, \mathrm{~T} ; \mathrm{H}^{1}\right)$ such that

$$
\begin{aligned}
& \phi_{l M} \stackrel{w}{\rightarrow} \phi \text { in } \mathrm{L}^{2}\left(0, \mathrm{~T} ; \mathrm{H}^{3}\right), \quad \phi_{l M} \quad \underline{w^{*}} \phi \text { in } \mathrm{L}^{\infty}\left(0, \mathrm{~T} ; \mathrm{H}^{1}\right), \\
& \overline{\mathbf{u}}_{l M} \stackrel{w}{\rightarrow} \mathbf{u} \text { in } \mathrm{L}^{2}\left(0, \mathrm{~T} ; \mathrm{H}^{1}\right), \quad \overline{\mathbf{u}}_{l M} \quad \underline{w} \mathbf{u} \text { in } \mathrm{L}^{\infty}\left(0, \mathrm{~T} ; \mathrm{L}^{2}\right), \\
& \partial_{\mathrm{t}} \phi_{l M} \stackrel{w}{\rightarrow} \partial_{\mathrm{t}} \phi \text { in } \mathrm{L}^{2}\left(0, \mathrm{~T} ; \mathrm{H}^{-3}\right) .
\end{aligned}
$$

Also, by applying an improvement of the Aubin compactness result in [21], we have

$$
\phi_{\mathrm{lM}} \rightarrow \phi \text { strongly in } \mathrm{L}^{2}\left(0, \mathrm{~T} ; \mathrm{H}^{2}\right) .
$$

Now we may pass to the limit, showing that the limit function $(\phi, \mathbf{u})$ is indeed a weak solution in the sense of (2.1). This completes the proof of Theorem 2.1. 


\section{Global in time strong solution}

The main results of this paper are the following global existence and uniqueness of solutions for the CHS system.

Theorem 3.1. Let $\phi_{0}=\phi(\cdot, 0) \in \mathrm{H}^{2}(\Omega)$. Then there exists a unique global (in time) strong solution for the dynamic CHS equation (1.2), (1.3), (1.4), such that for any $\mathrm{T}>0$

$$
\begin{aligned}
& \|\phi\|_{\mathrm{L}^{\infty}\left(0, \mathrm{~T} ; \mathrm{H}^{2}\right)} \leqslant \mathrm{B}_{1}, \quad\|\phi\|_{\mathrm{L}^{2}\left(0, \mathrm{~T} ; \mathrm{H}^{4}\right)} \leqslant \mathrm{B}_{2}, \quad\left\|\partial_{\mathrm{t}} \phi\right\|_{\mathrm{L}^{2}\left(0, \mathrm{~T} ; \mathrm{L}^{2}\right)} \leqslant \mathrm{B}_{3}, \\
& \|\mathbf{u}\|_{\mathrm{L}^{\infty}\left(0, \mathrm{~T} ; \mathrm{H}^{1}\right)} \leqslant \mathrm{B}_{4}, \quad\|\mathbf{u}\|_{\mathrm{L}^{2}\left(0, \mathrm{~T} ; \mathrm{H}^{2}\right)} \leqslant \mathrm{B}_{5},
\end{aligned}
$$

in which $\mathrm{B}_{1}$ and $\mathrm{B}_{4}$ are time independent and $\mathrm{B}_{2}, \mathrm{~B}_{3}$, and $\mathrm{B}_{5}$ are time dependent constants.

To prove this result, two important inequalities must be introduced.

Lemma 3.2 (Elliptic regularity [15]). There is a constant $C>0$, such that, for any $\psi \in \mathrm{H}^{\mathrm{m}+2}(\Omega)$,

$$
\begin{aligned}
& \|\psi\|_{\mathrm{H}^{\mathrm{m}+2}} \leqslant \mathrm{C}\left(\|\Delta \psi\|_{\mathrm{H}^{\mathrm{m}}}+\|\psi\|_{\mathrm{L}^{2}}\right), \\
& \|\psi\|_{\mathrm{H}^{2 \mathrm{~m}}} \leqslant \mathrm{C}\left(\left\|\Delta^{\mathrm{m}} \psi\right\|_{\mathrm{L}^{2}}+\|\psi\|_{\mathrm{L}^{2}}\right), \\
& \|\psi\|_{\mathrm{H}^{2 \mathrm{~m}+1}} \leqslant \mathrm{C}\left(\left\|\nabla \Delta^{\mathrm{m}} \psi\right\|_{\mathrm{L}^{2}}+\|\psi\|_{\mathrm{L}^{2}}\right) .
\end{aligned}
$$

Lemma 3.3 (Gagliardo-Nirenberg inequality [25]). Assume that $u \in \mathrm{L}^{\mathrm{q}}\left(\mathbb{R}^{\mathrm{n}}\right), \mathrm{D}^{\mathrm{m}} \mathrm{u} \in \mathrm{L}^{\mathrm{r}}\left(\mathbb{R}^{\mathrm{n}}\right), 1 \leqslant \mathrm{q}, \mathrm{r} \leqslant$ $\infty, 0 \leqslant j \leqslant m$. we have the estimate as follows

$$
\left\|D^{j} u\right\|_{L^{p}} \leqslant C\left\|D^{m} u\right\|_{L^{r}}^{\alpha}\|u\|_{L^{q}}^{1-\alpha},
$$

where $\mathrm{C}$ is a positive constant, $0 \leqslant \frac{j}{m} \leqslant \alpha \leqslant 1$,

$$
\frac{1}{p}=\frac{j}{n}+\alpha\left(\frac{1}{r}-\frac{m}{n}\right)+(1-\alpha) \frac{1}{q}
$$

Prior estimates for $\phi \in \mathrm{L}^{\infty}\left(0, \mathrm{~T} ; \mathrm{H}^{2}\right) \cap \mathrm{L}^{2}\left(0, \mathrm{~T} ; \mathrm{H}^{4}\right)$ and for $\boldsymbol{u} \in \mathrm{L}^{\infty}\left(0, \mathrm{~T} ; \mathrm{H}^{1}\right) \cap \mathrm{L}^{2}\left(0, \mathrm{~T} ; \mathrm{H}^{2}\right)$ are needed to establish a global in time strong solution. In particular, the estimate for the nonlinear convection term is vital in our proof.

3.1. Priori estimates for $\phi \in \mathrm{L}^{\infty}\left(0, \mathrm{~T} ; \mathrm{H}^{2}\right) \cap \mathrm{L}^{2}\left(0, \mathrm{~T} ; \mathrm{H}^{4}\right)$ and $\mathbf{u} \in \mathrm{L}^{\infty}\left(0, \mathrm{~T} ; \mathrm{H}^{1}\right) \cap \mathrm{L}^{2}\left(0, \mathrm{~T} ; \mathrm{H}^{2}\right)$

Taking the inner product of (1.2) with $2 \Delta^{2} \phi$ yields

$$
\frac{\mathrm{d}}{\mathrm{dt}}\|\Delta \phi\|^{2}+2 \epsilon^{2}\left\|\Delta^{2} \phi\right\|^{2}=2\left(\Delta\left(\phi^{3}\right), \Delta^{2} \phi\right)-2\left(\Delta \phi, \Delta^{2} \phi\right)-2\left(\mathbf{u} \cdot \nabla \phi, \Delta^{2} \phi\right) .
$$

The term associated with the concave diffusion can be treated in a straightforward way, with the help of a Sobolev interpolation inequality,

$$
-2\left(\Delta \phi, \Delta^{2} \phi\right)=2\|\nabla \Delta \phi\|^{2} \leqslant \mathrm{C}\left(\|\nabla \phi\|^{\frac{1}{3}} \cdot\left\|\Delta^{2} \phi\right\|^{\frac{2}{3}}\right)^{2} \leqslant \mathrm{C}\|\nabla \phi\|^{\frac{2}{3}} \cdot\left\|\Delta^{2} \phi\right\|^{\frac{4}{3}} \leqslant C A_{1}^{\frac{2}{3}} \cdot\left\|\Delta^{2} \phi\right\|^{\frac{4}{3}},
$$

in which the global in time $\mathrm{H}^{1}$ estimate (as shown in Theorem 2.1) was applied in the last step.

For the term associated with the cubic nonlinear part in (3.2), an application of Cauchy-Schwartz inequality yields,

$$
2\left(\Delta\left(\phi^{3}\right), \Delta^{2} \phi\right) \leqslant 2\left\|\Delta\left(\phi^{3}\right)\right\| \cdot\left\|\Delta^{2} \phi\right\|
$$

At the same time, it follows from the standard expansion

$$
\Delta\left(\phi^{3}\right)=3 \phi^{2} \Delta \phi+6 \phi|\nabla \phi|^{2},
$$


that

$$
\begin{aligned}
\left\|\Delta\left(\phi^{3}\right)\right\| & \leqslant 3 \mathrm{C}\|\phi\|_{\mathrm{L}^{6}}^{2} \cdot\|\Delta \phi\|_{\mathrm{L}^{6}}+6 \mathrm{C}\|\phi\|_{\mathrm{L}^{6}} \cdot\|\nabla \phi\|_{\mathrm{L}^{6}}^{2} \\
& \leqslant \mathrm{C}\left(\|\phi\|_{\mathrm{H}^{1}}^{2} \cdot\|\Delta \phi\|_{\mathrm{H}^{1}}+\|\phi\|_{\mathrm{H}^{1}} \cdot\|\nabla \phi\|_{\mathrm{H}^{1}}^{2}\right) \\
& \leqslant \mathrm{C}\left(A_{1}^{2} \cdot\|\Delta \phi\|_{\mathrm{H}^{1}}+\mathrm{A}_{1} \cdot\|\nabla \phi\|_{\mathrm{H}^{1}}^{2}\right) \\
& \leqslant \mathrm{C}\left(A_{1}^{2} \cdot\|\nabla \phi\|^{\frac{1}{3}} \cdot\left\|\Delta^{2} \phi\right\|^{\frac{2}{3}}+\mathrm{A}_{1} \cdot\left(\|\nabla \phi\|^{\frac{2}{3}} \cdot\left\|\Delta^{2} \phi\right\|^{\frac{1}{3}}\right)^{2}\right) \\
& \leqslant \mathrm{C} A_{1}^{\frac{7}{3}}\left\|\Delta^{2} \phi\right\|^{\frac{2}{3}}
\end{aligned}
$$

in which the 3-D Sobolev embedding from $\mathrm{H}^{1}$ into $\mathrm{L}^{6}$ was repeatedly applied in the derivation. Its combination with (3.4) results in

$$
2\left(\Delta\left(\phi^{3}\right), \Delta^{2} \phi\right) \leqslant C A_{1}^{\frac{7}{3}}\left\|\Delta^{2} \phi\right\|^{\frac{5}{3}} .
$$

For the term associated with the nonlinear convection, we also start with an application of Hölder inequality,

$$
-2\left(\mathbf{u} \cdot \nabla \phi, \Delta^{2} \phi\right) \leqslant 2\|\mathbf{u}\|_{L^{\infty}} \cdot\|\nabla \phi\| \cdot\left\|\Delta^{2} \phi\right\| \leqslant 2 A_{1}\|\mathbf{u}\|_{L^{\infty}} \cdot\left\|\Delta^{2} \phi\right\| .
$$

Meanwhile, a combination of the 3-D Sobolev embedding, interpolation inequality and elliptic regularity shows that

$$
\begin{aligned}
\|\mathbf{u}\|_{L^{\infty}} & \leqslant C_{\delta}\|\mathbf{u}\|_{H^{\frac{3}{2}+\delta}} \leqslant C_{\delta}\|\mathbf{u}\|^{\frac{1-2 \delta}{4}} \cdot\|\mathbf{u}\|_{H^{2}}^{\frac{3+2 \delta}{4}} \leqslant C_{\delta}\|\mathbf{u}\|^{\frac{1-2 \delta}{4}} \cdot(\|\mathbf{u}\|+\|\Delta \mathbf{u}\|)^{\frac{3+2 \delta}{4}} \\
& \leqslant C_{\delta} A_{4}^{\frac{1-2 \delta}{4}} \cdot\left(A_{4}+\|\Delta \mathbf{u}\|\right)^{\frac{3+2 \delta}{4}} \leqslant C_{\delta} A_{4}\left(1+\|\Delta \mathbf{u}\|^{\frac{3+2 \delta}{4}}\right) .
\end{aligned}
$$

As a result, a combination of (3.2), (3.3), (3.5), (3.6) and (3.7) implies that

$$
\frac{\mathrm{d}}{\mathrm{dt}}\|\Delta \phi\|^{2}+2 \epsilon^{2}\left\|\Delta^{2} \phi\right\|^{2} \leqslant \mathrm{C}\left(\mathrm{A}_{1}^{\frac{2}{3}}\left\|\Delta^{2} \phi\right\|^{\frac{4}{3}}+\mathrm{A}_{1}^{\frac{7}{3}}\left\|\Delta^{2} \phi\right\|^{\frac{5}{3}}+\mathrm{A}_{1} \mathrm{~A}_{4}\left(1+\|\Delta \mathbf{u}\|^{\frac{3+2 \delta}{4}}\right) \cdot\left\|\Delta^{2} \phi\right\|\right) .
$$

For (1.3), taking the inner product with $-2 \Delta \mathbf{u}$ yields

$$
\frac{\mathrm{d}}{\mathrm{dt}}\|\nabla \mathbf{u}\|^{2}+2\|\Delta \mathbf{u}\|^{2}+2\|\nabla \mathbf{u}\|^{2}=2 \gamma(\Delta \mathbf{u}, \phi \nabla \mu),
$$

in which the orthogonality between $\Delta \mathfrak{u}$ and $\nabla p$ comes from the incompressible property of $\Delta \mathfrak{u}$.

For the nonlinear term appearing on the right hand side, a preliminary estimate indicates that

$$
\begin{aligned}
\|\phi \nabla \mu\| & \leqslant\|\phi\|_{L^{\infty}} \cdot\left\|\nabla\left(\phi^{3}-\phi-\epsilon^{2} \Delta \phi\right)\right\| \\
& \leqslant\|\phi\|_{L^{\infty}}\left(\left\|\nabla\left(\phi^{3}\right)\right\|+\|\phi\|+\epsilon^{2}\|\nabla \Delta \phi\|\right) \\
& \leqslant\|\phi\|_{L^{\infty}}\left(\left\|\nabla\left(\phi^{3}\right)\right\|+A_{1}+\epsilon^{2}\|\nabla \Delta \phi\|\right) .
\end{aligned}
$$

The term $\|\phi\|_{L^{\infty}}$ can be bounded by

$$
\begin{aligned}
\|\phi\|_{\mathrm{L}^{\infty}} & \leqslant \mathrm{C}\left(\|\phi\|_{\mathrm{L}^{6}}^{\frac{3}{4}}\|\nabla \Delta \phi\|^{\frac{1}{4}}+\|\phi\|_{\mathrm{L}^{6}}\right) \leqslant \mathrm{C}\left(\|\phi\|_{\mathrm{H}^{1}}^{\frac{3}{4}}\|\nabla \Delta \phi\|^{\frac{1}{4}}+\|\phi\|_{\mathrm{H}^{1}}\right) \\
& \leqslant \mathrm{C}\left(\|\phi\|_{\mathrm{H}^{1}}^{\frac{3}{4}}\left(\|\nabla \phi\|^{\frac{1}{3}} \cdot\left\|\Delta^{2} \phi\right\|^{\frac{2}{3}}\right)^{\frac{1}{4}}+\|\phi\|_{\mathrm{H}^{1}}\right) \\
& \leqslant \mathrm{C}\left(A_{1}^{\frac{5}{6}} \cdot\left\|\Delta^{2} \phi\right\|^{\frac{1}{6}}+\mathrm{A}_{1}\right),
\end{aligned}
$$

with the help of Gagliardo-Nirenberg inequality. The two other terms in $\nabla \mu$ can be analyzed as

$$
\begin{aligned}
\left\|\nabla\left(\phi^{3}\right)\right\| & =\left\|3 \phi^{2} \nabla \phi\right\| \leqslant 3\|\phi\|_{\mathrm{L}^{6}}^{2} \cdot\|\nabla \phi\|_{\mathrm{L}^{6}} \leqslant \mathrm{C}\|\phi\|_{\mathrm{H}^{1}}^{2} \cdot\|\nabla \phi\|_{\mathrm{H}^{1}} \\
& \leqslant C A_{1}^{2} \cdot\|\nabla \phi\|^{\frac{2}{3}} \cdot\left\|\Delta^{2} \phi\right\|^{\frac{1}{3}} \leqslant C A_{1}^{\frac{8}{3}}\left\|\Delta^{2} \phi\right\|^{\frac{1}{3}} \\
\|\nabla \Delta \phi\| & \leqslant \mathrm{C}\|\nabla \phi\|^{\frac{1}{3}} \cdot\left\|\Delta^{2} \phi\right\|^{\frac{2}{3}} \leqslant \mathrm{CA}_{1}^{\frac{1}{3}} \cdot\left\|\Delta^{2} \phi\right\|^{\frac{2}{3}} .
\end{aligned}
$$


A substitution of (3.11), (3.12), (3.13) into (3.10) yields

$$
\|\phi \nabla \mu\| \leqslant \mathrm{C}\left(\mathrm{A}_{1}^{\frac{5}{6}} \cdot\left\|\Delta^{2} \phi\right\|^{\frac{1}{6}}+\mathrm{A}_{1}\right)\left(\mathrm{A}_{1}^{\frac{8}{3}} \cdot\left\|\Delta^{2} \phi\right\|^{\frac{2}{3}}+\mathrm{A}_{1}\right) \leqslant \mathrm{C} \mathrm{A}_{1}^{\frac{19}{6}} \cdot\left\|\Delta^{2} \phi\right\|^{\frac{5}{6}}+\mathrm{C}\left(\mathrm{A}_{1}\right) .
$$

Going back to (3.9), we arrive at

$$
\frac{\mathrm{d}}{\mathrm{dt}}\|\nabla \mathbf{u}\|^{2}+2\|\Delta \mathbf{u}\|^{2}+2\|\nabla \mathbf{u}\|^{2}=2 \gamma(\Delta \mathbf{u}, \phi \nabla \mu) \leqslant \mathrm{C} \gamma\left(\mathrm{A}_{1}^{\frac{19}{6}} \cdot\left\|\Delta^{2} \phi\right\|^{\frac{5}{6}}+\mathrm{C}\left(\mathrm{A}_{1}\right)\right) \cdot\|\Delta \mathbf{u}\| .
$$

Consequently, a combination of (3.8), and (3.14) implies that

$$
\begin{aligned}
\frac{\mathrm{d}}{\mathrm{dt}}\left(\|\Delta \phi\|^{2}\right. & \left.+\|\nabla \mathbf{u}\|^{2}\right)+2 \epsilon^{2}\left\|\Delta^{2} \phi\right\|^{2}+2\left(\|\Delta \mathbf{u}\|^{2}+\|\nabla \mathbf{u}\|^{2}\right) \\
\leqslant & C\left(A_{1}^{\frac{2}{3}}\left\|\Delta^{2} \phi\right\|^{\frac{4}{3}}+A_{1}^{\frac{7}{3}}\left\|\Delta^{2} \phi\right\|^{\frac{5}{3}}+A_{1} A_{4}\left(1+\|\Delta \mathbf{u}\|^{\frac{3+2 \delta}{4}}\right) \cdot\left\|\Delta^{2} \phi\right\|\right. \\
& \left.+\left(A_{1}^{\frac{19}{6}} \cdot\left\|\Delta^{2} \phi\right\|^{\frac{5}{6}}+C\left(A_{1}\right)\right) \cdot\|\Delta \mathbf{u}\|\right) .
\end{aligned}
$$

Since the power index sum for $\left\|\Delta^{2} \phi\right\|$ and $\|\Delta \mathbf{u}\|$ is less than 2 (in each term), the Young inequality $\left(a b \leqslant \frac{a^{p}}{p}+\frac{b^{q}}{q}\right.$ with $\left.\frac{1}{p}+\frac{1}{q}=1\right)$ can be applied to bound them:

$$
\begin{aligned}
& C A_{1}^{\frac{2}{3}}\left\|\Delta^{2} \phi\right\|^{\frac{4}{3}} \leqslant C_{\epsilon} A_{1}^{2}+\frac{\epsilon^{2}}{4}\left\|\Delta^{2} \phi\right\|^{2}, \quad\left(p=3, q=\frac{3}{2}\right), \\
& C A_{1}^{\frac{7}{3}}\left\|\Delta^{2} \phi\right\|^{\frac{5}{3}} \leqslant C_{\epsilon} A_{1}^{14}+\frac{\epsilon^{2}}{4}\left\|\Delta^{2} \phi\right\|^{2}, \quad\left(p=6, q=\frac{6}{5}\right), \\
& C A_{1} A_{4}\left\|\Delta^{2} \phi\right\| \leqslant C_{\epsilon} A_{1}^{2} A_{4}^{2}+\frac{\epsilon^{2}}{8}\left\|\Delta^{2} \phi\right\|^{2}, \quad(p=2, q=2), \\
& \mathrm{CA}_{1} \mathrm{~A}_{4}\|\Delta \mathbf{u}\|^{\frac{3+2 \delta}{4}} \cdot\left\|\Delta^{2} \phi\right\| \leqslant \mathrm{C}_{\epsilon, \mathrm{A}_{1}, \mathrm{~A}_{4}}+\frac{\epsilon^{2}}{8}\left\|\Delta^{2} \phi\right\|^{2}+\frac{1}{4}\|\Delta \mathbf{u}\|^{2}, \quad(\mathrm{p}=2, \mathrm{q}=2), \\
& \mathrm{CA}_{1}^{\frac{19}{6}}\left\|\Delta^{2} \phi\right\|^{\frac{5}{6}} \cdot\|\Delta \mathbf{u}\| \leqslant \mathrm{C}_{\epsilon, \mathrm{A}_{1}}+\frac{\epsilon^{2}}{4}\left\|\Delta^{2} \phi\right\|^{2}+\frac{1}{4}\|\Delta \mathbf{u}\|^{2}, \quad(\mathrm{p}=2, \mathrm{q}=2), \\
& C\left(A_{1}\right) \cdot\|\Delta \mathbf{u}\| \leqslant C\left(A_{1}\right)+\frac{1}{4}\|\Delta \mathbf{u}\|^{2}, \quad(p=2, q=2) \text {. }
\end{aligned}
$$

Therefore,

$$
\frac{\mathrm{d}}{\mathrm{dt}}\left(\|\Delta \phi\|^{2}+\|\nabla \mathbf{u}\|^{2}\right)+\epsilon^{2}\left\|\Delta^{2} \phi\right\|^{2}+\|\Delta \mathbf{u}\|^{2}+\|\nabla \mathbf{u}\|^{2} \leqslant \mathrm{C}_{\mathrm{A}_{1}, \mathrm{~A}_{4}, \epsilon} .
$$

Moreover, with an application of elliptic regularity,

$$
C_{2}\|\Delta \phi\|^{2} \leqslant\left\|\Delta^{2} \phi\right\|^{2}, \quad C_{2}\|\nabla \mathbf{u}\|^{2} \leqslant\|\Delta \mathbf{u}\|^{2},
$$

we get

$$
\frac{\mathrm{d}}{\mathrm{dt}}\left(\|\Delta \phi\|^{2}+\|\nabla \mathbf{u}\|^{2}\right)+\mathrm{C}_{2} \epsilon^{2}\left(\|\Delta \phi\|^{2}+\|\nabla \mathbf{u}\|^{2}\right) \leqslant \mathrm{C}_{\mathrm{A}_{1}, \mathrm{~A}_{4}, \epsilon} .
$$

This in turn gives an estimate containing an exponential decay for $\phi \in \mathrm{H}^{2}$ and $\mathbf{u} \in \mathrm{H}^{1}$ :

$$
\|\Delta \phi(\mathrm{t})\|^{2}+\|\nabla \mathbf{u}(\mathrm{t})\|^{2} \leqslant \mathrm{e}^{-\mathrm{C}_{2} \epsilon^{2} \mathrm{t}}\left(\|\Delta \phi(0)\|^{2}+\|\nabla \mathbf{u}(0)\|^{2}\right)+\frac{\mathrm{C}_{\mathrm{A}_{1}, \mathrm{~A}_{4}, \epsilon}}{\mathrm{C}_{2} \epsilon^{2}}:=\mathrm{B}_{1}^{*} .
$$

Hence, a uniform in time $\mathrm{H}^{2}$ bound for $\phi$ is obtained by the elliptic regularity,

$$
\|\phi(t)\|_{H^{2}} \leqslant C(\|\phi(t)\|+\|\triangle \phi(t)\|) \leqslant C\left(A_{1}+\left(B_{1}^{*}\right)^{1 / 2}\right):=B_{1}
$$


in which clearly $B_{1}$ is time independent, and

$$
\|\mathbf{u}\|_{\mathrm{H}^{1}} \leqslant \mathrm{C}(\|\mathbf{u}\|+\|\nabla \mathbf{u}\|) \leqslant \mathrm{C}\left(\mathrm{A}_{4}+\left(\mathrm{B}_{1}^{*}\right)^{1 / 2}\right):=\mathrm{B}_{4},
$$

where $\mathrm{B}_{4}$ is also time independent.

Based on (3.15), the following estimate can also be derived,

$$
\int_{0}^{T}\left(\left\|\Delta^{2} \phi\right\|^{2}+\|\Delta \mathbf{u}\|^{2}\right) d t \leqslant \frac{C_{A_{1}, A_{4}, \epsilon} T+\|\Delta \phi(t=0)\|^{2}+\|\nabla \mathbf{u}(t=0)\|^{2}}{\epsilon^{2}}=B_{2}^{*} .
$$

Consequently, due to the elliptic regularity $\|\phi(t)\|_{H^{4}} \leqslant C\left(\|\phi(t)\|+\left\|\triangle^{2} \phi(t)\right\|\right)$, an $L^{2}\left(0, T ; H^{4}\right)$ estimate for $\phi$ is available,

$$
\|\phi\|_{\mathrm{L}^{2}\left(0, \mathrm{~T} ; \mathrm{H}^{4}\right)} \leqslant \mathrm{C}\left(\|\phi(\mathrm{t})\|_{\mathrm{L}^{2}\left(0, \mathrm{~T} ; \mathrm{L}^{2}\right)}+\left\|\triangle^{2} \phi(\mathrm{t})\right\|_{\mathrm{L}^{2}\left(0, \mathrm{~T} ; \mathrm{L}^{2}\right)}\right) \leqslant \mathrm{C}\left(\mathrm{A}_{2}+\left(\mathrm{B}_{2}^{*}\right)^{1 / 2}\right):=\mathrm{B}_{2} .
$$

Also, by the elliptic regularity $\|\mathbf{u}\|_{H^{2}} \leqslant \mathrm{C}(\|\mathbf{u}\|+\|\Delta \mathbf{u}\|)$, we can obtain the estimate for $\mathbf{u}$,

$$
\|\mathbf{u}\|_{\mathrm{L}^{2}\left(0, \mathrm{~T} ; \mathrm{H}^{2}\right)} \leqslant \mathrm{C}\left(\|\mathbf{u}\|_{\mathrm{L}^{2}\left(0, \mathrm{~T} ; \mathrm{L}^{2}\right)}+\|\Delta \mathbf{u}\|_{\mathrm{L}^{2}\left(0, \mathrm{~T} ; \mathrm{L}^{2}\right)}\right) \leqslant \mathrm{C}\left(\mathrm{A}_{5}+\left(\mathrm{B}_{2}^{*}\right)^{1 / 2}\right):=\mathrm{B}_{5} .
$$

Finally, from CHS equation (1.2), an $\mathrm{L}^{2}\left(0, \mathrm{~T} ; \mathrm{L}^{2}\right)$ estimate for $\partial_{t} \phi$ can be obtained

$$
\left\|\partial_{\mathrm{t}} \phi\right\|_{\mathrm{L}^{2}\left(0, \mathrm{~T} ; \mathrm{L}^{2}\right)} \leqslant \mathrm{C}\left(\mathrm{A}_{1}\|\mathbf{u}\|_{\mathrm{L}^{2}\left(0, \mathrm{~T} ; \mathrm{H}^{2}\right)}+\left\|\triangle\left(\phi^{3}\right)\right\|_{\mathrm{L}^{2}\left(0, \mathrm{~T} ; \mathrm{L}^{2}\right.}+\mathrm{B}_{2}\right) \leqslant \mathrm{C}\left(\mathrm{A}_{1} \mathrm{~B}_{4}+\mathrm{B}_{1}^{3} \mathrm{~T}+\mathrm{B}_{2}\right):=\mathrm{B}_{3},
$$

with a Sobolev analysis applied for $\triangle\left(\phi^{3}\right)$ in the last step.

\subsection{Existence of the strong solution}

The proof of existence in Theorem 3.1. Since all these estimates are at hand, a global in time strong solution for the CHS equation can be established by the standard Galerkin procedure. Taking the inner product with (2.3) by $2 \triangle^{2} \phi_{M}$, we have

$$
\begin{aligned}
\frac{\mathrm{d}}{\mathrm{dt}}\left\|\triangle \phi_{M}\right\|^{2}+2 \varepsilon^{2}\left\|\triangle^{2} \phi_{M}\right\|^{2}= & 2\left(\triangle\left(\mathcal{P}_{M}\left(\phi_{M}^{3}\right)\right), \triangle^{2} \phi_{M}\right)-2\left(\triangle \phi_{M}, \triangle^{2} \phi_{M}\right) \\
& -2\left(\mathcal{P}_{M}(\overline{\mathbf{u}} \cdot \nabla \phi), \triangle^{2} \phi_{M}\right) .
\end{aligned}
$$

The prior analysis provided above can be applied to (3.16) in the same way, and we are able to get the following bounds, uniform in $M>1$,

$$
\begin{aligned}
& \left\|\phi_{M}\right\|_{L^{\infty}\left(0, T ; H^{2}\right)} \leqslant B_{1}, \quad\left\|\phi_{M}\right\|_{L^{2}\left(0, T ; H^{4}\right)} \leqslant B_{2}, \quad\left\|\partial_{t} \phi_{M}\right\|_{L^{2}\left(0, T ; L^{2}\right)} \leqslant B_{3}, \\
& \left\|\overline{\mathbf{u}}_{M}\right\|_{L^{\infty}\left(0, T ; H^{1}\right)} \leqslant B_{4}, \quad\left\|\overline{\mathbf{u}}_{M}\right\|_{L^{2}\left(0, T ; H^{2}\right)} \leqslant B_{5},
\end{aligned}
$$

and details are left to interested readers.

As a result, the limit function $(\phi, \mathbf{u})$ is a strong solution to the CHS equation (1.2), (1.3), (1.4). Hence the proof of existence in Theorem 3.1 is complete and the estimates (3.1) are available.

\subsection{Uniqueness of the strong solution}

Assume $\left(\phi_{1}, \mathbf{u}_{1}\right)$ and $\left(\phi_{2}, \mathbf{u}_{2}\right)$ are two strong solutions to (1.2), (1.3), (1.4), with the same initial data, that is,

$$
\begin{aligned}
& \partial_{\mathrm{t}} \phi_{1}+\mathbf{u}_{1} \cdot \nabla \phi_{1}=\triangle\left(\phi_{1}^{3}-\phi_{1}-\varepsilon^{2} \triangle \phi_{1}\right), \\
& \partial_{\mathrm{t}} \mathbf{u}_{1}-\triangle \mathbf{u}_{1}+\mathbf{u}_{1}=-\gamma \mathcal{P}_{\mathrm{H}}\left(\phi_{1} \nabla\left(\phi_{1}^{3}-\phi_{1}-\varepsilon^{2} \triangle \phi_{1}\right)\right), \\
& \partial_{\mathrm{t}} \phi_{2}+\mathbf{u}_{2} \cdot \nabla \phi_{2}=\triangle\left(\phi_{2}^{3}-\phi_{2}-\varepsilon^{2} \triangle \phi_{2}\right), \\
& \partial_{\mathrm{t}} \mathbf{u}_{2}-\triangle \mathbf{u}_{2}+\mathbf{u}_{2}=-\gamma \mathcal{P}_{\mathrm{H}}\left(\phi_{2} \nabla\left(\phi_{2}^{3}-\phi_{2}-\varepsilon^{2} \triangle \phi_{2}\right)\right), \\
& \left.\phi_{1}\right|_{\mathrm{t}=0}=\left.\phi_{2}\right|_{\mathrm{t}=0}=\phi_{0} \in \mathrm{H}^{2},
\end{aligned}
$$


in which $\left(\phi_{i}, \mathbf{u}_{i}\right), i=1,2$ satisfy

$$
\begin{aligned}
& \left\|\phi_{i}\right\|_{\mathrm{L}^{\infty}\left(0, \mathrm{~T} ; \mathrm{H}^{2}\right)} \leqslant \mathrm{B}_{1}, \quad\left\|\phi_{i}\right\|_{\mathrm{L}^{2}\left(0, \mathrm{~T} ; \mathrm{H}^{4}\right)} \leqslant \mathrm{B}_{2}, \quad\left\|\partial_{\mathrm{t}} \phi_{i}\right\|_{\mathrm{L}^{2}\left(0, \mathrm{~T} ; \mathrm{L}^{2}\right)} \leqslant \mathrm{B}_{3}, \\
& \left\|\mathbf{u}_{\mathrm{i}}\right\|_{\mathrm{L}^{\infty}\left(0, \mathrm{~T} ; \mathrm{H}^{1}\right)} \leqslant \mathrm{B}_{4}, \quad\left\|\mathbf{u}_{\mathrm{i}}\right\|_{\mathrm{L}^{2}\left(0, \mathrm{~T} ; \mathrm{H}^{2}\right)} \leqslant \mathrm{B}_{5} .
\end{aligned}
$$

The difference functions are given by

$$
\tilde{\phi}=\phi_{1}-\phi_{2}, \quad \tilde{\mathbf{u}}=\mathbf{u}_{1}-\mathbf{u}_{2}
$$

Subtracting (3.19)-(3.20) from (3.17)-(3.18), we get

$$
\begin{aligned}
\partial_{\mathrm{t}} \tilde{\phi}+\mathbf{u}_{1} \cdot \nabla \tilde{\phi}= & \triangle\left(\left(\phi_{1}^{2}+\phi_{1} \phi_{2}+\phi_{2}^{2}\right) \tilde{\phi}-\tilde{\phi}-\varepsilon^{2} \triangle \tilde{\phi}\right), \\
\partial_{\mathrm{t}} \tilde{\mathbf{u}}-\triangle \tilde{\mathbf{u}}+\tilde{\mathbf{u}}= & -\gamma \mathcal{P}_{\mathrm{H}}\left(\phi_{2} \nabla\left(\left(\phi_{1}^{2}+\phi_{1} \phi_{2}+\phi_{2}^{2}\right) \tilde{\phi}-\tilde{\phi}-\varepsilon^{2} \triangle \tilde{\phi}\right)\right. \\
& \left.+\tilde{\phi} \nabla\left(\phi_{1}^{3}-\phi_{1}-\varepsilon^{2} \triangle^{2} \phi_{1}\right)\right), \\
\left.\tilde{\phi}\right|_{\mathrm{t}=0}= & 0 .
\end{aligned}
$$

We also introduce the following lemma to prove the uniqueness of the global in time strong solution. For the details, see [5].

Lemma 3.4 ([5]). The following estimates are valid.

$$
\begin{aligned}
& \int_{\Omega} \tilde{\phi}(\mathrm{t}) \mathrm{d} x=0, \quad \forall \mathrm{t}>0, \\
& \|\tilde{\phi}\| \leqslant \mathrm{C}\|\nabla \tilde{\phi}\|, \\
& \left\|\nabla\left(\left(\phi_{1}^{2}+\phi_{1} \phi_{2}+\phi_{2}\right) \tilde{\phi}\right)\right\| \leqslant \mathrm{CB}_{1}^{2}\|\nabla \tilde{\phi}\|, \\
& \int_{0}^{T} \mathrm{M}_{1}^{2}(\mathrm{t}) \mathrm{d} \mathrm{t} \leqslant \mathrm{CB}_{5}^{2}, \quad \mathrm{M}_{1}(\mathrm{t})=\left\|\mathbf{u}_{1}(\mathrm{t})\right\|_{\mathrm{L}^{\infty}}, \\
& \int_{0}^{T} \mathrm{M}_{2}^{4}(\mathrm{t}) \mathrm{dt} \leqslant \mathrm{B}_{1}^{2} \mathrm{~B}_{2}^{2}, \quad \mathrm{M}_{2}(\mathrm{t})=\left\|\nabla \triangle \phi_{1}(\mathrm{t})\right\|, \\
& \left\|\tilde{\phi} \nabla\left(\phi_{1}^{3}-\phi_{1}-\varepsilon^{2} \triangle \phi_{1}\right)\right\| \leqslant \mathrm{C}_{\mathrm{B}_{1}}\|\nabla \tilde{\phi}\|+\mathrm{CM}_{2}(\mathrm{t})\|\nabla \tilde{\phi}\|^{3 / 4} \cdot\|\nabla \triangle \tilde{\phi}\|^{1 / 4},
\end{aligned}
$$

in which $\mathrm{B}_{1}$ and $\mathrm{B}_{4}$ are time independent and $\mathrm{B}_{2}, \mathrm{~B}_{3}$, and $\mathrm{B}_{5}$ are time dependent constants.

Next, we present the following uniqueness analysis in Theorem 3.1.

The proof of the uniqueness in Theorem 3.1. Taking the inner product with (3.21) by $-2 \triangle \tilde{\phi}$, we get

$$
\begin{aligned}
& \frac{\mathrm{d}}{\mathrm{dt}}\|\nabla \tilde{\phi}\|^{2}+ 2 \varepsilon^{2}\|\nabla \triangle \tilde{\phi}\|^{2} \\
&=-2\left(\mathbf{u}_{1} \tilde{\phi}+\tilde{\mathbf{u}} \phi_{2}, \nabla \triangle \tilde{\phi}\right)+2\|\triangle \tilde{\phi}\|^{2}+2\left(\nabla\left(\left(\phi_{1}^{2}+\phi_{1} \phi_{2}+\phi_{2}^{2}\right) \tilde{\phi}\right), \nabla \triangle \tilde{\phi}\right) .
\end{aligned}
$$

The $\|\triangle \tilde{\phi}\|^{2}$ can be controlled with the help of Young inequality,

$$
\|\triangle \tilde{\phi}\|^{2}=-(\nabla \tilde{\phi}, \nabla \triangle \tilde{\phi}) \leqslant\|\nabla \tilde{\phi}\| \cdot\|\nabla \triangle \tilde{\phi}\| \leqslant 2 \varepsilon^{-2}\|\nabla \tilde{\phi}\|^{2}+\frac{\varepsilon^{2}}{8}\|\nabla \triangle \tilde{\phi}\|^{2} .
$$

According to the formula (3.24) in Lemma 3.4, the last nonlinear term in (3.26) can also be analyzed by a similar method as follows,

$$
\begin{aligned}
\left(\nabla\left(\left(\phi_{1}^{2}+\phi_{1} \phi_{2}+\phi_{2}^{2}\right) \tilde{\phi}\right), \nabla \triangle \tilde{\phi}\right) & \leqslant\left\|\nabla\left(\left(\phi_{1}^{2}+\phi_{1} \phi_{2}+\phi_{2}^{2}\right) \tilde{\phi}\right)\right\| \cdot\|\nabla \triangle \tilde{\phi}\| \\
& \leqslant \mathrm{CB}_{1}^{2}\|\nabla \tilde{\phi}\| \cdot\|\nabla \triangle \tilde{\phi}\| \\
& \leqslant \mathrm{CB}_{1}^{4} \varepsilon^{-2}\|\nabla \tilde{\phi}\|^{2}+\frac{\varepsilon^{2}}{8}\|\nabla \triangle \tilde{\phi}\|^{2}
\end{aligned}
$$


Considering the nonlinear terms associated with the velocity convection, we first discuss the simpler one,

$$
\begin{aligned}
-\left(\mathbf{u}_{1} \tilde{\phi}, \nabla \triangle \tilde{\phi}\right) & \leqslant\left\|\mathbf{u}_{1}\right\|_{\mathrm{L}^{\infty}} \cdot\|\tilde{\phi}\| \cdot\|\nabla \triangle \tilde{\phi}\|=\mathrm{CM}_{1}(\mathrm{t})\|\tilde{\phi}\| \cdot\|\nabla \triangle \tilde{\phi}\| \\
& \leqslant 2 \mathrm{CM}_{1}^{2}(\mathbf{t}) \varepsilon^{-2}\|\nabla \tilde{\phi}\|^{2}+\frac{\varepsilon^{2}}{8}\|\nabla \triangle \tilde{\phi}\|^{2} .
\end{aligned}
$$

On the other hand, for the other nonlinear term in the convection, from (3.22), we can rewrite the form of $\phi_{2} \nabla \triangle \tilde{\phi}$ as follows,

with

$$
\phi_{2} \nabla \triangle \tilde{\phi}=\frac{1}{\gamma \mathcal{\varepsilon}^{2}}\left(\left(\partial_{\mathrm{t}} \tilde{\mathbf{u}}-\triangle \tilde{\mathbf{u}}+\tilde{\mathbf{u}}\right)+\mathcal{N} \mathcal{L} \mathcal{E}\right)+\nabla \varphi^{\prime}
$$

$$
\mathcal{N} \mathcal{L} \mathcal{E}=\gamma \phi_{2} \nabla\left(\tilde{\phi}\left(\phi_{1}^{2}+\phi_{1} \phi_{2}+\phi_{2}^{2}\right)-\tilde{\phi}\right)+\tilde{\phi} \nabla\left(\phi_{1}^{3}-\phi_{1}-\varepsilon^{2} \triangle \phi_{1}\right)
$$

Therefore we have the following estimate,

$$
\begin{aligned}
-\left(\tilde{\mathbf{u}} \phi_{2}, \nabla \triangle \tilde{\phi}\right) & =-\left(\tilde{\mathbf{u}}, \phi_{2} \nabla \triangle \tilde{\phi}\right)=-\frac{1}{\gamma \varepsilon^{2}}\left(\left(\tilde{\mathbf{u}}, \partial_{\mathrm{t}} \tilde{\mathbf{u}}-\triangle \tilde{\mathbf{u}}+\tilde{\mathbf{u}}\right)+(\tilde{\mathbf{u}}, \mathcal{N} \mathcal{L} \mathcal{E})\right), \\
& =-\frac{1}{\gamma \varepsilon^{2}}\left(\frac{1}{2} \frac{\mathrm{d}}{\mathrm{dt}}\|\tilde{\mathbf{u}}\|^{2}+\|\nabla \tilde{\mathbf{u}}\|^{2}+\|\tilde{\mathbf{u}}\|^{2}\right)-\frac{1}{\gamma \mathcal{E}^{2}}(\tilde{\mathbf{u}}, \mathcal{N} \mathcal{L} \mathcal{E}) \\
& \leqslant-\frac{1}{\gamma \varepsilon^{2}}\left(\frac{1}{2} \frac{\mathrm{d}}{\mathrm{dt}}\|\tilde{\mathbf{u}}\|^{2}+\|\nabla \tilde{\mathbf{u}}\|^{2}+\|\tilde{\mathbf{u}}\|^{2}\right)+\frac{1}{\gamma \mathcal{E}^{2}}\|\tilde{\mathbf{u}}\| \cdot\|\mathcal{N} \mathcal{L} \mathcal{E}\| \\
& \leqslant-\frac{1}{\gamma \varepsilon^{2}}\left(\frac{1}{2} \frac{\mathrm{d}}{\mathrm{dt}}\|\tilde{\mathbf{u}}\|^{2}+\|\nabla \tilde{\mathbf{u}}\|^{2}+\|\tilde{\mathbf{u}}\|^{2}\right)+\frac{1}{2 \gamma \mathcal{E}^{2}}\left(\|\tilde{\mathbf{u}}\|^{2}+\|\mathcal{N} \mathcal{L} \mathcal{E}\|^{2}\right),
\end{aligned}
$$

in which $\left(\tilde{\mathbf{u}}, \nabla \varphi^{\prime}\right)$ vanishes because of the property of the Helmholtz projection $\mathcal{P}_{H}$.

With formulas (3.23) and (3.25) from Lemma 3.4, the term $\|\mathcal{N} \mathcal{L} \mathcal{E}\|$ can also be derived,

$$
\begin{aligned}
\|\mathcal{N} \mathcal{L} \mathcal{E}\| & \leqslant\left\|\phi_{2} \nabla\left(\tilde{\phi}\left(\phi_{1}^{2}+\phi_{1} \phi_{2}+\phi_{2}^{2}\right)\right)\right\|+\left\|\tilde{\phi} \nabla\left(\phi_{1}^{3}-\phi_{1}-\varepsilon^{2} \triangle \phi_{1}\right)\right\|+\left\|\phi_{2} \nabla \tilde{\phi}\right\| \\
& \leqslant \mathrm{C}_{\mathrm{B}_{1}}\|\nabla \tilde{\phi}\|+\mathrm{CM}_{2}(\mathrm{t})\|\nabla \tilde{\phi}\|^{3 / 4}\|\nabla \triangle \tilde{\phi}\|^{1 / 4}+\left\|\phi_{2}\right\|_{\mathrm{L}^{\infty}} \cdot\|\nabla \tilde{\phi}\| \\
& \leqslant \mathrm{C}_{\mathrm{B}_{1}}\|\nabla \tilde{\phi}\|+\mathrm{CM}_{2}(\mathrm{t})\|\nabla \tilde{\phi}\|^{3 / 4}\|\nabla \triangle \tilde{\phi}\|^{1 / 4}
\end{aligned}
$$

which yields

$$
\begin{aligned}
\|\mathcal{N} \mathcal{L} \mathcal{E}\|^{2} & \leqslant \mathrm{C}_{\mathrm{B}_{1}}\|\nabla \tilde{\phi}\|^{2}+\mathrm{CM}_{2}(\mathrm{t})\|\nabla \tilde{\phi}\|^{3 / 2}\|\nabla \triangle \tilde{\phi}\|^{1 / 2} \\
& \leqslant \mathrm{C}_{\mathrm{B}_{1}}\|\nabla \tilde{\phi}\|^{2}+\mathrm{C}_{\gamma, \varepsilon} \mathrm{M}_{2}^{4 / 3}(\mathrm{t})\|\nabla \tilde{\phi}\|^{2}+\frac{\varepsilon^{2}}{4}\|\nabla \triangle \tilde{\phi}\|^{2} \\
& \leqslant\left(\mathrm{C}_{\mathrm{B}_{1}}+\mathrm{C}_{\gamma, \varepsilon} \mathrm{M}_{2}^{4 / 3}(\mathrm{t})\right)\|\nabla \tilde{\phi}\|^{2}+\frac{\gamma \varepsilon^{2}}{4}\|\nabla \triangle \tilde{\phi}\|^{2} .
\end{aligned}
$$

Consequently, a combination of (3.26), (3.27), (3.28), (3.29) and (3.30) leads to

$$
\begin{aligned}
\frac{\mathrm{d}}{\mathrm{d} t}\|\nabla \tilde{\phi}\|^{2}+ & \frac{1}{\gamma \mathcal{\varepsilon}^{2}} \frac{\mathrm{d}}{\mathrm{dt}}\|\tilde{\mathbf{u}}\|^{2}+\varepsilon^{2}\|\nabla \triangle \tilde{\phi}\|^{2} \\
\leqslant & 4 \varepsilon^{-2}\|\nabla \tilde{\phi}\|^{2}+2 \mathrm{~B}_{1}^{4} \varepsilon^{-2}\|\nabla \tilde{\phi}\|^{2}+4 \mathrm{M}_{1}^{2}(\mathrm{t}) \varepsilon^{-2}\|\nabla \tilde{\phi}\|^{2} \\
& +\left(\mathrm{C}_{\mathrm{B}_{1}}+\mathrm{C}_{\gamma, \varepsilon} \mathrm{M}_{2}^{4 / 3}(\mathrm{t})\right)\|\nabla \tilde{\phi}\|^{2}-\frac{1}{\gamma \varepsilon^{2}}\|\tilde{\mathbf{u}}\|^{2} .
\end{aligned}
$$

We denote $D(t, \gamma, \varepsilon)=4 \varepsilon^{-2}+2 B_{1}^{4} \varepsilon^{-2}+4 M_{1}^{2}(t) \varepsilon^{-2}+\left(C_{B_{1}}+C_{\gamma, \varepsilon} M_{2}^{4 / 3}(t)\right)$, and obtain the integrability of the coefficient,

$$
\begin{aligned}
\int_{0}^{T} D(t, \gamma, \varepsilon) d t & =\int_{0}^{T} 4 \varepsilon^{-2}+2 B_{1}^{4} \varepsilon^{-2}+4 M_{1}^{2}(t) \varepsilon^{-2}+\left(C_{B_{1}}+C_{\gamma, \varepsilon} M_{2}^{4 / 3}(t)\right) d t \\
& \leqslant C_{B_{1}, \varepsilon} T+C_{B_{1}, \varepsilon} B_{5}^{2}+C_{\gamma, \varepsilon} \int_{0}^{T}\left(M_{2}^{4}(t)+1\right) d t \\
& \leqslant C_{B_{1}, \varepsilon} T+C_{B_{1}, \varepsilon} B_{5}^{2}+C_{\gamma, \varepsilon} B_{1}^{2} B_{2}^{2}:=E_{T} .
\end{aligned}
$$


Furthermore, the inequality (3.31) can be rewritten as

$$
\frac{\mathrm{d}}{\mathrm{dt}}\|\nabla \tilde{\phi}\|^{2}+\frac{1}{\gamma \varepsilon^{2}} \frac{\mathrm{d}}{\mathrm{dt}}\|\tilde{\mathbf{u}}\|^{2} \leqslant \mathrm{D}(\mathrm{t}, \gamma, \varepsilon)\left(\|\nabla \tilde{\phi}\|^{2}+\|\tilde{\mathbf{u}}\|^{2}\right) .
$$

Therefore, an application of the Gronwall-Bellman inequality to (3.32) implies that

$$
\|\nabla \tilde{\phi}(t)\|^{2}+\frac{1}{\gamma \varepsilon^{2}}\|\tilde{\mathbf{u}}(\mathrm{t})\|^{2} \leqslant \exp \left(E_{\mathrm{T}}\right)\left(\|\nabla \tilde{\phi}(0)\|^{2}+\frac{1}{\gamma \varepsilon^{2}}\|\tilde{\mathbf{u}}(0)\|^{2}\right)=0,
$$

with the trivial initial data applied in the last step. By the inequality $\|\tilde{\phi}\| \leqslant C\|\nabla \tilde{\phi}\|$, the uniqueness of the strong solution $(\phi, \mathbf{u})$ is assured. The proof of the uniqueness in Theorem 3.1 is complete.

Remark 3.5. Similarly, the techniques proposed in this paper can be applied to analyze the higher order derivatives of the solution of the dynamic CHS equation in the same fashion, (for example, the initial function $\left.\phi_{0}=\phi(\cdot, 0) \in \mathrm{H}^{\mathrm{m}}(\Omega),(\mathrm{m} \geqslant 3)\right)$, and the details are skipped for brevity.

\section{Acknowledgment}

The authors are very grateful to reviewers for carefully reading this paper and their comments. We also greatly appreciate many helpful discussions with Cheng Wang (University of Massachusetts, Dartmouth), in particular for their insightful suggestions, and the support provided for this paper by the Science and Technology Department of Sichuan Province in China (No. 2017GZ0316).

\section{References}

[1] N. Alikakos, P. Bates, G. Fusco, Slow motion for the Cahn-Hilliard equation in one space dimension, J. Differential Equations, 90 (1991), 81-135. 1

[2] P. Bates, P. Fife, The dynamics of nucleation for the Cahn-Hilliard equation, SIAM J. Appl. Math., 53 (1993), 990-1008. 1

[3] J. W. Cahn, J. E. Hilliard, Free energy of a nonuniform system. I. Interfacial free energy, J. Chem. Phys., 28 (1958), 258-267. 1

[4] W. Chen, W. Feng, Y. Liu, C. Wang, S. Wise, A Second Order Energy Stable Scheme for the Cahn-Hilliard-Hele-Shaw Equations, arXiv, 2016 (2016), 34 pages. 1

[5] W. Chen, Y. Liu, C. Wang, S. M. Wise, Global-in-time smooth solution to a three-dimensional Cahn-Hilliard-Stokes model, (submitted). 1, 3.3, 3.4

[6] K. Cheng, C. Wang, S. M. Wise, X. Yue, A second-order, weakly energy-stable pseudo-spectral scheme for the CahnHilliard equation and its solution by the homogeneous linear iteration method, J. Sci. Comput., 69 (2016), 1083-1114. 1

[7] A. E. Diegel, C. Wang, S. M. Wise, Stability and convergence of a second-order mixed finite element method for the Cahn-Hilliard equation, IMA J. Numer. Anal., 36 (2016), 1867-1897.

[8] C. Elliott, H. Garcke, On the Cahn-Hilliard equation with degenerate mobility, SIAM J. Math. Anal., 27 (1996), $404-423$. 1

[9] X. Feng, Fully discrete finite element approximations of the Navier-Stokes-Cahn-Hilliard diffuse interface model for twophase fluids, SIAM J. Numer. Anal., 40 (2006), 1049-1072. 1

[10] X. Feng, O. A. Karakashian, Fully discrete dynamic mesh discontinous Galerkin methods for the Cahn-Hilliard equation of phase transition, Math. Comp., 76 (2007), 1093-1117. 1

[11] X. Feng, A. Prohl, Error analysis of a mixed finite element method for the Cahn-Hilliard equation, Numer. Math., 99 (2004), 47-84. 1

[12] X. Feng, S. M. Wise, Analysis of a Darcy-Cahn-Hilliard diffuse interface model for the Hele-Shaw flow and its fully discrete finite element approximation, SIAM J. Numer. Anal., 50 (2012), 1320-1343. 1, 1

[13] S. Frigeri, M. Grasselli, Nonlocal Cahn-Hilliard-Navier-Stokes systems with singular potentials, Dyn. Partial Differ. Equ., 9 (2012), 273-304. 1

[14] C. G. Gal, M. Grasselli, Asymptotic behavior of a Cahn-Hilliard-Navier-Stokes system in 2D, Ann. Inst. H. Poincaré Anal. Non Linéaire, 27 (2010), 401-436. 1

[15] D. Gilbarg, N. Trudinger, Elliptic Partial Differential equations of Second Order, Springer-Verlag, Berlin, (1983). 3.2

[16] Y. He, Y. Liu, Stability and convergence of the spectral Galerkin method for the Cahn-Hilliard equation, Numer. Meth. Partial Diff. Eq., 24 (2008), 1485-1500. 1 
[17] K. Kang, J. Kim, J. Lowengrub, Conservative multigrid methods for Cahn-Hilliard fluids, J. Comput. Phys., 193 (2004), 511-543.

[18] D. Kay, R. Welford, A multigrid finite element solver for the Cahn-Hilliard equation, SIAM J. Sci. Comput., 29 (2006), 288-304. 1

[19] D. Kay, R. Welford, Efficient numerical solution of Cahn-Hilliard-Navier-Stokes fluids in 2D, SIAM J. Sci. Comput., 29 (2007), 2241-2257. 1

[20] J. Lowengrub, E. S. Titi, K. Zhao, Analysis of a mixture model of tumor growth, Eur. J. Appl. Math., 24 (2013), 691-734. 1

[21] R. Temam, Navier-Stokes Equations: Theory and Numerical Analysis, North-Holland Publishing Co., New York, (1977). 2.3

[22] X. Wang, H. Wu, Long-time behavior of the Hele-Shaw-Cahn-Hilliard system, Asymptotic Anal., 78 (2012), 217-245. 1, 1

[23] X. Wang, Z. Zhang, Well-posedness of the Hele-Shaw-Cahn-Hilliard system, Ann. Inst. H. Poincaré Anal. Non Linéaire, 30 (2013), 367-384. 1

[24] S. Wise, Unconditionally stable finite difference, nonlinear multigrid simulation of the Cahn-Hilliard-Hele-Shaw system of equations, J. Sci. Comput., 44 (2010), 38-68. 1

[25] S. You, X. Ning, Well-posedness for a class of generalized Zakharov system, J. Nonlinear Sci. Appl., 10 (2017), 1289-1302 3.3

[26] K. Zhao, Large time behavior of a Cahn-Hilliard-Boussinesq system on a bounded domain, Electron. J. Differ. Eq., 2011 (2011), 21 pages. 1 